\title{
Effects of morphological complexity on the P600 response in L2 speakers of English
}

\author{
Trevor McAllister-Day \\ Department of Linguistics \\ University of Washington \\ Seattle \\ June 9, 2017 \\ Supervisor: \\ Lee Osterhout, $\mathrm{PhD}$ \\ Professor, Department of Psychology \\ Adjunct Professor, Department of Linguistics \\ University of Washington \\ Seattle
}





\section{Contents}

Contents iii

$1 \quad$ Introduction $\ldots \ldots \ldots \ldots \ldots \ldots \ldots$

1.1 Electroencephalography . . . . . . . . . . . 2

1.2 ERPs and L1 Processing . . . . . . . . . . . . 2

1.3 The P600 and Morphological Complexity . . . . . . . . 5

$1.4 \quad$ ERPs and L2 Processing . . . . . . . . . . . . 7

1.5 The Present Study . . . . . . . . . . . . . . . . . 9

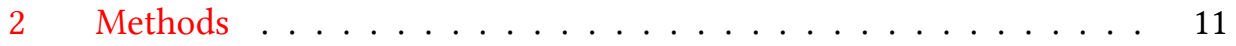

$2.1 \quad$ Participants . . . . . . . . . . . . . . . 11

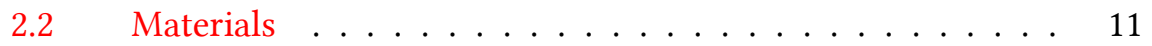

$2.3 \quad$ Procedure . . . . . . . . . . . . . . . 12

2.4 Data acquisition and analysis . . . . . . . . . . . . . . . 13

$3 \quad$ Results . . . . . . . . . . . . . . . . . . . 13

3.1 Behavioral Data . . . . . . . . . . . . . . 13

3.2 Proficiency Scores . . . . . . . . . . . . . . . . . 14

$3.3 \quad$ ERP Findings . . . . . . . . . . . . . . . . . . . 15

$3.4 \quad$ Complexity Results . . . . . . . . . . . . . . . . . . . 19

$4 \quad$ Discussion . . . . . . . . . . . . . . . . . . . . . 19

4.1 Complexity Results . . . . . . . . . . . . . . . 19

4.2 Trends . . . . . . . . . . . . . . . . 21

4.3 Limitations . . . . . . . . . . . . . . . 21

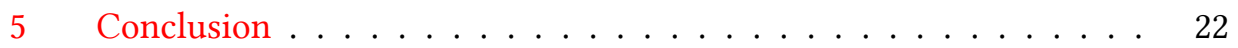

$\begin{array}{ll}\text { References } & 23\end{array}$ 



\begin{abstract}
Native speakers of English are differentially sensitive to morphologically simple and morphologically complex when both are ungrammatical. A larger P600 is elicited by sentences like *"The dogs must running" than *"The dog is run." This is taken to mean that native speakers have an easier time processing the second condition, either because the base form of the verb is underspecified for a number of featural specifications, or because predictions in "be" contexts are weaker than predictions in modal conditions. We find that L1 speakers of Mandarin who speak English as a second language show a P600 to ungrammatical, morphologically complex critical verbs, but no signal at all to ungrammatical, morphologically simple critical verbs. This suggests they lack the information to form predictions about "be" contexts or have an oversimplified rule about present participles: namely, that they only appear in "be" contexts, without realizing that auxiliary "be" licenses only present participles.
\end{abstract}





\section{Introduction}

Humanity is characterized by the possession of language faculty. Not only are we the only species with such a capability, every person acquires a first language when exposed from birth, regardless of variation in input quality. Language processing is fast, accurate, and automatic. We also observe that individuals are capable of learning second languages (L2), where the age of acquisition (AoA) is generally negatively correlated with ultimate attainment. The idea that languages can only be learned imperfectly after a certain age (usually puberty) is known the critical period hypothesis [1]. However, elements of language are differentially sensitive to age. Judgments regarding L2 syntactic rules can be affected by delays in exposure as short as 1 to 3 years of age, while judgments regarding semantic anomalies are only affected by much longer delays, between 11 to 16 years of age [2]. Likewise, production of L2 is also rated as more accented by native speakers as AoA increases [3].

Behavioral evidence from studies such as these suggest that L2 acquisition is not solely governed by a single critical period, but rather by a system of sensitive periods for units like phonology, syntax, and semantics. However, measures of linguistic performance do not examine the neural underpinnings of the difference between first language (L1) and L2 processing, and how L2 processing changes as proficiency increases.

Some neural circuitry is shared between L1 and L2 processing. Functional magnetic resonance imaging (fMRI) has provided evidence that the majority of regions activated during language processing are shared between L1 and L2, with a few differences. In a review paper, Kotz [4] summarizes the similarities and differences: The left middle frontal gyrus was activated similarly in both L1 and L2 [5, 6], while the inferior frontal gyrus was recruited in L2 speakers [5, 7, 8]. Additionally, the left superior temporal gyrus is less activated in L2 processing [8, 9], suggesting a trade-off between the two areas.

However, in evaluating just how similar L1 and L2 processing are, fMRI is somewhat limited, as it has a temporal resolution of seconds, which is inadequate for assessing online language processing, which happens at the millisecond level. On the other hand, electroencephalography (EEG) has a much higher temporal resolution, and can be used to investigate rapid processing events in the brain. The rest of this section will provide an introduction to EEG and event-related potential (ERP) technique, how they have been used to learn about L1/L2 processing, and explain how the current study might provide more insight into how similar L1 and L2 processing can be. 


\subsection{Electroencephalography}

EEG records neural processing by monitoring minute voltage fluctuations within neurons and across synapses of the brain. Voltage readings are obtained by electrodes placed against the scalp. The changes in voltage in a single neuron are much too small to be read, therefore, EEG picks up on simultaneous activity across thousands or millions of neurons acting together, presumably as part of the same processing event. Notably, the surface location from which the EEG signal is strongest does not reflect the location of the relevant neurons in the brain, but rather, the direction in which they are oriented (see Steinhauer [10] for a review).

However, raw EEG signals are highly noisy, and when taken alone, are not informative of a single processing event (linguistic or otherwise). ERPs are created by averaging EEG signals across multiple trials of the same type, which makes them an effective tool for analyzing EEG data. ERP techniques allow for the detection of underlying signals by averaging out random noise caused by factors such as electrical signals generated by muscle movements like eye blinks, other processing events, and non-physiologic interference from nearby electronics [11].

\subsection{ERPs and First-Language Processing}

Prior research has identified several ERPs that are robustly sensitive to aspects of real-time language comprehension. They, in the order of onset, are: the left-anterior negativity (LAN) and early LAN (ELAN), the N00, and the P600. Typical waveforms of these three components can be seen in Figure 1. These components have been used to study many facets of processing of ungrammatical sentences [12, 13], and changes in processing of an L2 as proficiency changes [14].

\section{The LAN}

The left-anterior negativity (LAN) occurs within $100 \mathrm{~ms}$ to $500 \mathrm{~ms}$ of the critical word onset, and primarily correlates with morphosyntactic errors. Within the LAN, the very early LAN (ELAN) correlates with word-category errors [15], and has been proposed to be part of a family of ERP effects related to syntactic, morphological, or working memory issues [4].

However, Friederici's originally study dealt with auditory modalities, and most reading studies fail to observe an ELAN, finding instead only a later anterior negativity (post-300 ms), an N400 or a relative positivity in the relevant time range (see Steinhauer and Drury [16] for a review; cf. Tanner et al. [17] and Tanner, Nicol, and Brehm [18]). Because of their lack of robustness, the ELAN and LAN will not be considered for this study. 
(a)

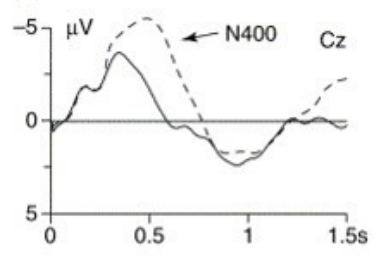

- Das Hemd wurde bebügelt. The shirt was 'ironed".

- Das Gewitter wurde gebügelt. (b)
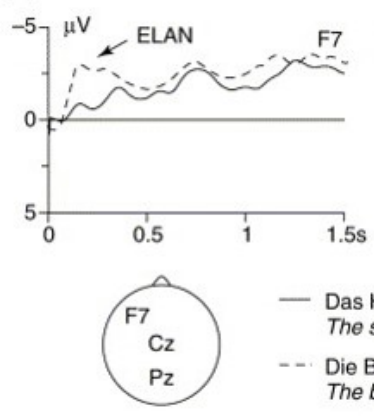

(c)

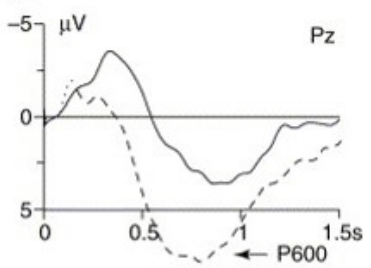

- Das Hemd wurde bebūgelt. The shirt was 'ironed'.

- - Die Bluse wurde am gebūgelt The blouse was on ironed.

Figure 1: The three language-related ERP components: (a) N400; (b) ELAN; and (c) P600. Signals are averaged over the three electrodes presented below the waveforms. Duplicated from Friederici [15]

\section{The N400}

The N400 is a negative-going wave that peaks at $400 \mathrm{~ms}$ post-stimulus. It is largest over centro-parietal sites, with a slight right-hemisphere bias for written stimuli [19, 20]. Usually used to study context and semantic fit, the N400 is elicited by semantic or pragmatic violations, such as the mismatch between "dog" and "coffee" in (1) [19].

${ }^{*}$ I take my coffee with cream and $d o g$.

The N400 is sensitive to effects of frequency, predictability, and priming. The amplitude of the N400 component is highly correlated $(r=.9)$ with the probability that the target word would appear in that context. This probability is known as cloze probability, which measures what percentage of individuals would insert that word into the sentence when given a blank. The N400 component can also be affected by semantic priming effects. In trials where the target was unrelated to a prime, the N400 was larger in amplitude than trials where the target was related to the prime [20].

\section{The P600}

In contrast to the N400, the P600 effect is elicited by structural violations such as grammatical agreement, tense, case, and verb subcategorization [12, 18, 21-27]. The P600 is a positive-going wave that begins roughly $500 \mathrm{~ms}$ after the presentation of an ungrammatical target word and lasts for several hundred milliseconds, peaking around $600 \mathrm{~ms}$ (hence the name). It is localized over the centro-parietal region [15]. 
Example 2) below is an example of a tense violation of the kind that elicits a P600 component [19].

(2) * The cats won't eating the food that Mary leaves them.

Additionally, the P600 has been used to study harmonic incongruities in music $[28,29]$, suggesting that the P600 reflects cognitive action common to language and music comprehension.

Past research has shown that the P600 effect is generated by syntactic or structural errors of various types, as in (3a-3f) (duplicated from Osterhout, McLaughlin, and Bersick [19]), in addition to the aforementioned tense errors like (2).

(3) a. Phrase structure anomalies:

* "The scientist criticized Max's of proof the theorem."

b. Verb subcategorization anomalies:

* "The broker persuaded to sell the stock."

c. Sentence-constituent movement anomalies:

* "I wonder which dress the guests at the part were shocked when the bride wore."

d. Subject-verb number disagreement:

* "The elected officials hopes to succeed."

e. Reflexive-antecedent number disagreement:

* "The hungry guests helped himself to the meal."

f. Reflexive-antecedent gender disagreement:

* "The successful woman congratulated himself on the promotion."

Notably, the P600 effect also appears during the processing of temporary ambiguities introduced by unclear garden-path sentences, such as the ambiguity between (4a) and (4b) at "The broker persuaded" [30]. It is unclear until more context is added whether "persuaded" is the main verb of the sentence (as it is in (4a)) or part of a relative clause (as in (4b)).

(4) a. [NP The broker ] persuaded the man [ ${ }_{\mathrm{CP}}$ to sell the stock ].

b. [NP The broker [CP persuaded to sell the stock ] ] was sent to jail.

Research such as this suggests the P600 component is not tied solely to error processing, but rather, reflects the difficulty of processing. As well as parsing of garden-path sentences, the $\mathrm{P} 600$ also appears during successful syntactic processing of difficult-to-integrate phenomena such as resolution of long-distance dependencies $[31,32]$ and ambiguity detection $[33,34]$. While both (5a) and (5b) are grammatical, P600 effects appear in sentences such as (5a), which is more difficult to process 
than (5b), due to the effort required to resolve the gap created by WH-movement of "who."

(5) a. Emily wondered who the performer in the concert had imitated for the audience's amusement.

b. Emily wondered whether the performer in the concert had imitated a pop star for the audience's amusement

Additionally, in both sentences, the NP "performer in the concert" is linked to the agent thematic role assigned by "imitated." However, (5a) is also more difficult to process because a second stage of integration must occur between the verb and "who," which receives a second thematic role of patient. This additional integration is also more challenging because the "who" phrase is separated from the verb by a number of words [31].

To summarize, the $\mathrm{P} 600$ component is related to processing ungrammatical stimuli as well as grammatical but complex stimuli. As will be discussed in The P600 and Morphological Complexity, the P600 can vary in amplitude. When it does, higher amplitudes are taken to be indicative of more difficult processing [14, 31].

\subsection{P600 Interactions with Morphological Complexity}

While the P600 is well-established, it is unclear how the P600 component is affected by properties of the stimuli and/or cognitive tasks. One early paper, Osterhout and Nicol [22], demonstrates the problem, in comparing responses elicited by grammatical stimuli such as (6a) to ungrammatical stimuli such as (6b) (critical word italicized).

(6) a. The cat will eat the food.

b. * The cat will eating the food.

Here, the critical word (eat/eating) is subject to two conditions: grammaticality and morphological complexity: "Eating" is morphologically complex, but "eat" is not. This confound makes it difficult to isolate whether the difference found between conditions is due only to ungrammaticality, or whether they may be affected by the interaction of ungrammaticality and complexity.

Mehravari et al. [14] tackles this issue by holding the morphological form of the critical word constant and modifying the the pre-critical context to generate grammatical and ungrammatical sentences. For example, (7a) and (7b) below.

(7) a. The cat was eating ...

b. * The cat will eating ... 
In separating the effects of morphological complexity and grammaticality, Mehravari et al. [14] found that morphological complexity alone did not elicit a P600 response, but the authors found that the P600 was larger for morphologically complex verbs than morphologically simple ones when both were ungrammatical. This finding has consequences for the theory of the underlying processing. Clearly, the subjects (English monolinguals) do not process morphologically complex and morphologically simple verbs in the same manner. Two theories might account for the findings: The first theory suggests a difference in the strength of predictions made as comprehension advances. The second theory suggests P600 components are a result of feature mismatches.

(Theory 1) One theoretical explanation holds that critical words displaying features predicted by context do not trigger processing difficulties; but words displaying unexpected features trigger difficulties reflected by the P600. To summarize this model, more difficult processing should result from having to overcome stronger initial predictions. Speakers may construct predictions based on knowledge of the cooccurrence frequency of certain morphological forms. The stimuli used in Mehravari et al. [14] and here comprised two pre-verbal conditions: finite forms of be (am, is, are, was, were) or modal auxiliary verbs (can, could, shall, should, will, would, must, might, may).

In an analysis of the Corpus of Contemporary American English [35], a participial form of a verb followed a finite form of be 13.5 percent of the time (the grammatical condition) and the base form of a verb followed a modal 34.6 percent of the time (the grammatical condition). This means, for a native speaker, a mistake in a sentence with a modal pre-verbal condition is a violation of a relatively stronger prediction than the prediction made in a sentence with a finite be pre-verbal condition. Because errors in modal sentences were formed by making the target verb morphologically complex, it is unclear whether the increase in P600 amplitude is due to predictions formed when the pre-verbal was introduced, or related to the morphological structure of the target verb.

However, in the context of learners, results may be confounded by the fact there is a different rate of acquisition of knowledge about be and modals. Given the reasonable assumption that forms of be are more common in English than modals, learners will have unequal exposure to each. Participial forms are one of the first forms acquired by learners of English [36], suggesting learners may be able to form predictions about $b e$, but not have enough data to form predictions about modal behavior.

Under this model, the absolute and relative amplitude of learner P600 components may reveal the strength of L2 speakers' predictions. If subjects are proficient enough, then a difference in amplitude will show they have also acquired knowledge and make predictions about the frequency of such structures in English, while simi- 
lar amplitudes will reveal that completely native-like processing is not yet available to them.

(Theory 2) A second contributor to P600 amplitude is cue overlap between retrieval probes and potential targets. To be available as a target, the cues on an item must match, at least partially, the cues available in working memory. The P600 is therefore implicated when a search for the licenser of an ungrammatical target turns up empty. In the context of morphological complexity, verb base forms are underspecified for a number of features, suggesting that they may not trigger a P600 even when ungrammatical, because few featural mismatches are present to trigger a backward search.

The question then remains under this model whether L2 speakers have an knowledge of verbal features advanced enough to initiate a backward search when there exists no obvious licenser. The lack of a P600 might indicate that either they misidentify the features of the cue or target, or during online processing, when encountering a feature mismatch, they may conclude that their earlier identification was mistaken and continue processing with the assumption that the later cue is correct, in order to process the sentence in time.

\subsection{ERPs and Second-Language Processing}

The N400 and P600 pattern differently in L2 processing compared to L1 processing. The N400 has been reported to be elicited by semantic errors in L2 speakers, albeit temporally delayed relative to target onset [2,37-41] and reduced in amplitude [39] in L2 speakers. On the other hand, the P600 is less robust. In lower-proficiency learners the P600 component is reduced and delayed [41], or missing altogether [42, 43], especially when AoA is greater than 16, but has been shown to appear in highproficiency speakers with an AoA between 1 and 11 years [2].

However, the study that found a P600 in early learners (Rossi et al. [41]) studied native speakers of Italian learning German (and vice versa), two languages with more morphosyntactic similarities than the languages studied by Hahne and Friederici [42] (German and Russian), which found no P600 at a similar stage. This raises the possibility that L1 affects a learner's sensitivity to the morphosyntax of L2, an assertion supported by a study on L1 Japanese learners of English, which found no P600s at either the low- or high-proficiency level [40]. Likewise, Sabourin and Stowe [44], in a study on the acquisition of L2 Dutch grammatical gender found that German L1 learners showed a P600 effect to gender violations, while learners with a Romance language as L1 did not. In a similar study, Frenck-Mestre et al. [45] demonstrated that the acquisition of L2 French grammatical gender is similarly affected by L1. However, L1 German learners did not show a P600 response to French concord errors, whereas L1 English learners did, a finding the authors suggest is due to the 
interaction of German gender and plurality, in which gender is neutralized in DP concord. In the long term, studies have found that the ERPs generated by highproficiency late bilinguals are not distinguishable from native speakers in certain morphosyntactic tasks (in an artifical grammar-learning study) [46, 47] (cf. [10]), indicating native-like processing is theoretically ultimately attainable.

McLaughlin et al. [43] provides an overview of three L2 learning studies; one studying English-speaking learners of German, one studying English-speaking learners of French, and one studying English-speaking learners of Finnish. In the German study, a cross-sectional study, learners were studied after one year and three years of university instruction. There was no relationship between hours of instruction and N400 and P600 effect sizes, nor was there a relationship between d-prime score and N400, but the relationship between d-prime score and the P600 effect was significant $(r=.53)$.

The findings of the German study suggest that less competent learners show strong N400 effects and more competent ones, P600 effects; however, interpretation is constrained by the cross-sectional design. This concern was addressed in a longitudinal study of English speakers learning French, specifically examining two rules: verbal agreement, which is similar to English, and gender concord, which is not. After three weeks of classroom instruction, learners showed N400 responses to verbal agreement anomalies (a rule similar to English), small differences after 16 weeks, and a P600 effect after 26 weeks. Further examination shows that the overall shift was driven by the existence of two subgroups of learners: one subgroup displaying primarily N400 effects and the other P600 effects. As in German, The effect size of of the P600, but not N400, was significantly positively correlated with d-prime score. However, for the dissimilar rule, learners' brain responses did not change between the grammatical and ungrammatical condition, and did not change over the course of instruction.

The third study, on Finnish vowel harmony, found similar results. In Finnish vowel harmony, if the initial vowel of a word is a front vowel $(/ y, ø, æ /)$, all subsequent vowels must be front or neutral vowels (/i, e/). If the initial vowel is a back vowel $(/ \mathrm{u}, \mathrm{o}, \mathrm{a} /)$, the subsequent vowels must be neutral or back. Native Finnish speakers show P600 effects to violations of vowel harmony. The learners showed a similar pattern to the German and French studies. At the first session, vowel harmony violations elicited reliable N400 effects. At the second session, learners were divided between showing N400 effects and P600 effects. At session 3, violations elicited a robust P600. Again, P600 effect sizes were significantly positively correlated with d-prime scores.

While morphological segmentation processes are fast and automatic in L1 processing, they are less so in L2 processing. The general move from an N400 to P600 reflects an underlying shift from reliance on lexicosemantic systems to morphosyn- 
tactic systems to process morphosyntactic anomalies [48, 49]. The findings detailed above suggest that learners begin relying on lexicosemantic systems when they are relatively new to the language, and shift to morphosyntactic systems based on a number of factors, including rule similarity and competence. Evidence from L1/L2 similarity studies suggests that learners grammaticalize features of L2 that are similar to L1 more quickly than dissimilar features.

In the section above, the presence of two distinct amplitudes for the P600 component are taken to be indicative of a native speaker's ability to rely on their knowledge of their L1 to process verbs based specifically on their knowledge of (1) word frequencies and/or (2) featural specifications. Mehravari et al. [14] shows that native speakers may find some violations less challenging to process than others. I hypothesize that learners will find all violations equally easy to process at first, before possibly being able to acquire enough knowledge of English to have weaker responses to some categories of violations. In terms of morphological complexity, I hypothesize that English learners will show P600s equal in amplitude to both kinds of violation (bare verb vs. present participle) before showing a smaller P600 to the bare verb condition, as native speakers of English do. However, considering the precritical context differs between morphological conditions (forms of "be" vs modals), it is perfectly possible learners may acquire knowledge of "be" violations and adapt to them before acquiring knowledge of modal violations at all.

\subsection{The Present Study}

As was discussed previously, different L1s can affect ERP results. Thus, for this study, it is important to control the L1 of the subjects. One accessible group of L2 English speakers is the L1 Mandarin student population, many of whom are recent emigrants from China or Taiwan, or are living only temporarily in the United States. International students from non-English-speaking countries attending the University of Washington are required to take either the Test of English as a Foreign Language, Internet-based and receive a score of 76 (out of 120) or the International English Language Testing System test and receive a 6.0 (out of 9.0) ${ }^{1}$.

Mandarin is also advantageous as an L1 for investigating how the P600 changes over time because it is highly morphosyntactically dissimilar to English. Although English morphology is relatively impoverished, Mandarin is even more so, and with the majority of verbal inflection being carried out by a system of particles. This means L1 Mandarin learners of English are relatively naïve to English verbal morphology as a grammatical feature, and should naturally progress through the complete path of grammaticalization of English inflection, including, presumably, the

\footnotetext{
${ }^{1}$ https://admit. washington.edu/apply/freshman/how-to-apply/international
} 
elicitation of an $\mathrm{N} 400$ to verb inflection violations, then the proposed "plain" P600, then finally a complex P600.

I predict that L2 English speakers who are competent enough in English to attend an English-speaking university will show P600s to structural problems, however, only the most advanced will know English well enough to have an easier time processing monomorphemic ungrammatical compared to morphological complexity ungrammatical verbs.

Regardless of the underlying models that affect the processing of ungrammatical morphologically complex verbs, this study investigates how difficult non-native speakers of English find the processing of ungrammatical and morphological complexity verbs. There are four possible findings of this study with regards to variation in P600 amplitude:

1. It is possible that they will find both types of verb equally as challenging to process as native speakers find morphologically complex verbs. In ERP terms, this means that they would have P600s equal in amplitude, and similar to the magnitude of native speaker morphological complexity-elicited P600s. This finding would suggest that learners of a certain ability are not proficient enough to rely on knowledge of frequency or featural specifications to use alternative, easier pathways for underspecified verbs.

2. Alternatively, they could find both verbs as equally as easy to process as native speakers find morphologically simple verbs. In ERP terms, the P600s would be equal in amplitude but similar in magnitude to native speaker morphologically simple verbs. This finding would suggest that learners of a certain ability do not consider frequency or featural specifications, and may simply skip over mismatches or inflectional affixes to speed processing and understanding.

3. Another possible result is that L2 speakers at all levels may show unequal, native-like P600 amplitudes, demonstrating that the presence of a P600 necessitates the availability of distinct pathways for underspecified and specified verbal forms.

4. A fourth possibility is that speakers may find morphologically simple verbs harder to process than morphologically complex verbs. A possible explanation for this finding would be that speakers are not knowledgeable enough about morphologically complex verbs to process them at all; but are knowledgeable enough about simple ones to process them and identify ungrammatical uses.

With a set of subjects with differential skill levels, unequal P600 amplitudes may appear in the more advanced speakers, but not the less-advanced, mirroring the shift from N400 to P600 found in early learners. This may aid to pinpoint the time frame 
at which learners acquire knowledge of verb form frequencies and/or verb feature specifications. Like Mehravari et al. [14], this experiment will not be able to identify whether form frequencies or feature specifications are responsible for underlying processing differences, if any are found.

\section{Methods}

\subsection{Participants}

Participants were 13 second-language speakers of English attending college at the University of Washington. All participants were age 18 or older, and the average age was 19.58 ( $\mathrm{SD}=1.24)$. Of the subjects, 4 were female (self-reported gender). Participants provided written informed consent and received course extra credit for taking part in the study. All experimental procedures were approved by the University of Washington Institutional Review Board.

Participants were required to speak Mandarin from birth and speak English nonnatively. The average age of exposure to English was 6.5 (min: 2.5; max: 10). One subject reported being exposed to English from birth, however, proficiency and frequency scores did not reflect a person who was consistently exposed to English in the home. Two participants spoke a language other than Mandarin natively ${ }^{2}$, however all subjects included in the analysis were Mandarin-dominant (as measured by self-reported proficiency and frequency measures). Multilingual subjects varied in whether they were more proficient in English or a third language.

All participants were not born in the United States. Ten were born in China, the other three were born in Japan, Malaysia, and Taiwan. Average age of arrival (AoA) was 16.08 (SD = 3.93). All but one subject are temporarily living in the U.S. to attend university.

\subsection{Materials}

The stimuli were identical to the stimuli used in Mehravari et al. [14]. They were 120 sentence quadruplets in a fully crossed $2 \times 2$ design (complexity $\times$ grammaticality). Sentences were either grammatical or the critical verb had a violation of tense morphology, and critical verbs were either morphologically simple (e.g. "run") or morphologically complex (e.g "running"). See Table 1 for an example quadruplet. All critical words were in either the base form or present participial form. The stimuli used 120 different verbs, chosen such that the written word form log-frequency of the base and present participial form was not significantly different for all 120

\footnotetext{
${ }^{2}$ Japanese, Bahasa (Indonesia)
} 


\begin{tabular}{lll}
\hline Grammaticality & Complexity & Sentence \\
\hline Grammatical & Simple & The dog must run to catch the ball. \\
Grammatical & Complex & The dog was running to catch the ball. \\
Ungrammatical & Simple & ${ }^{*}$ The dog was run to catch the ball. \\
Ungrammatical & Complex & ${ }^{*}$ The dog must running to catch the ball. \\
\hline
\end{tabular}

Table 1: Example experimental stimuli. Critical word in bold.

verbs (base form average frequency $=1.233$, present participle form average frequency $=1.230, t=0.067, p=0.946$ ), as provided in the CELEX2 database [50].

The word preceding the critical word was either a past-tense form of be (was or were) or a modal verb (should, could, would, will, can, must, may). The four versions of each sentence were distributed across four experimental lists, so that each participant saw only one version of each sentence. Each list contained an additional 60 grammatical filler sentences. Each list of 180 sentences was randomized and divided into three blocks of 60 sentences.

\subsection{Procedure}

Participants were tested in a single two-hour session. During preparation of the electrode cap, subjects filled out a number of self-assessments, including a language history questionnaire, an English self-rated proficiency questionnaire, and a handedness questionnaire based on the Edinburgh Handedness Inventory. After completion of the self-assessments, the participants took the Transparent Language English Proficiency Test ${ }^{3}$, a freely available online proficiency test.

Each participant was pseudorandomized to one of the four experimental lists. Participants in a comfortable recliner in front of a CRT monitor. They were instructed to relax and minimize movement and eye blinks while silently reading each sentence. Each sentence was presented in the following manner:

1. Blank screen

(1000 ms)

2. Fixation cross

(a) Interstimulus interval

3. Single word

(a) Interstimulus interval

4. Repeat (3)

\footnotetext{
${ }^{3}$ http://www .transparent.com/learn-english/proficiency-test.html
} 
5. Blank screen

(1000 ms)

6. Yes/no prompt

(no limit)

After the final blank screen, the participants were presented with a "yes/no" prompt, at which point they were instructed to provide an acceptability judgment, where sentences that were correct in every way received a "yes," and sentences which contained a grammatical and/or semantic violation received a "no." The "yes/no" prompt remained on screen until the participants responded. They were not instructed to respond as quickly as possible. During ERP studies, subjects are instructed not to blink, and are given inter-sentence breaks to blink. As soon as "yes" or "no" was chosen, presentation of the next sentence began. Participants were pseudorandomly assigned to use their left or right hand for the "yes" response.

\subsection{Data acquisition and analysis}

Continuous EEG was recorded from 19 tin electrodes in accordance with the 10-20 system [51], attached to an elastic cap (Electro-cap International). Eye movements and blinks were monitored by two electrodes: one beneath the left eye and one to the right of the right eye. An electrode on the left mastoid served as a reference. EEG was also recorded from an electrode placed on the right mastoid. An SAI bioamplifier system amplified EEG signals with a bandpass of $0.01 \mathrm{~Hz}$ to $40 \mathrm{~Hz}$ $(-3 \mathrm{~dB}$ cutoff). Waveforms were filtered offline below $30 \mathrm{~Hz}$. Impedances at scalp and mastoid electrodes were held below $5 \mathrm{k} \Omega$, and eye electrodes below $10 \mathrm{k} \Omega$.

Continuous analog-to-digital conversion of raw EEG data and stimulus triggers was perfromed at a sampling frequency of $200 \mathrm{~Hz}$. Data windows were time-locked to the onset of the critical word in each sentence (the bold words in Table 1), and averaged offline for each participant at each electrode in each experimental condition. Trials were removed due to contamination such as eye blinks, excessive muscle artifacts, or amplified blocking. Of all trials, 999 were rejected. In accordance with Mehravari et al. [14] and previous literature, the time windows of $300 \mathrm{~ms}$ to $500 \mathrm{~ms}$ and $500 \mathrm{~ms}$ to $900 \mathrm{~ms}$ were used to examine N400 and P600 effects, respectively.

\section{Results}

\subsection{Behavioral Data}

Results from the end-of-sentence judgment task showed non-native speakers are competent at grammaticality judgements, at least in the grammatical condition. Average overall, between-conditions accuracy was $66 \%(\mathrm{SD}=26)$. Table 2 lists the average accuracies by condition. 


\begin{tabular}{lrr}
\hline Condition & Mean & SD \\
\hline Grammatical, simple & 0.829 & 0.147 \\
Ungrammatical, simple & 0.564 & 0.248 \\
Grammatical, complex & 0.739 & 0.168 \\
Ungrammatical, complex & 0.519 & 0.314 \\
\hline
\end{tabular}

Table 2: Mean accuracies and standard deviation by condition

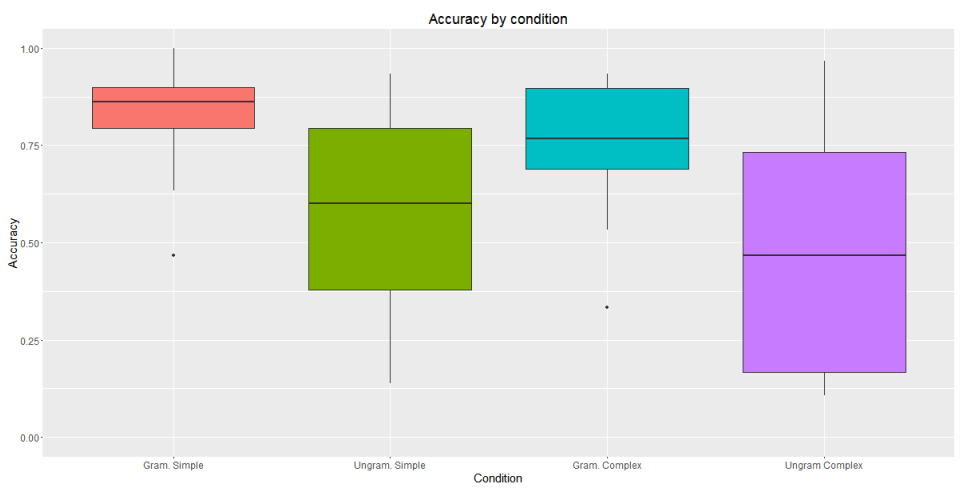

Figure 2: Accuracies by condition

A two-way between-subjects ANOVA was conducted to compare the effects of grammaticality and complexity on accuracy. The effect of complexity was not significant $(F(1)=1.151, p=0.288)$, but there was a significant effect of grammaticality $(F(1)=14.8, p<.001)$. As can be seen in Figure 2, variability was much higher in the ungrammatical condition than the grammatical condition. In other words, non-native speakers have a harder and inconsistent time correctly identifying ungrammatical sentences as wrong over identifying grammatical sentences as correct. However, morphological complexity did not affect their ability to identify sentences correctly.

\subsection{Proficiency Scores}

Participants were asked to rate their current proficiency on a 7-point Likert scale across multiple domains: pronunciation, grammar, vocabulary, reading comprehension, spoken comprehension, writing skills, spelling ability, and speaking ability.

We wanted to know how well self-rated proficiency correlated with the objective measurement of the TLEPT test. The average English self-assessment score (across subjects) was $4.96(\mathrm{SD}=.889)$, and the average TLEPT score was $84 \%(\mathrm{SD}=5.23)$. 


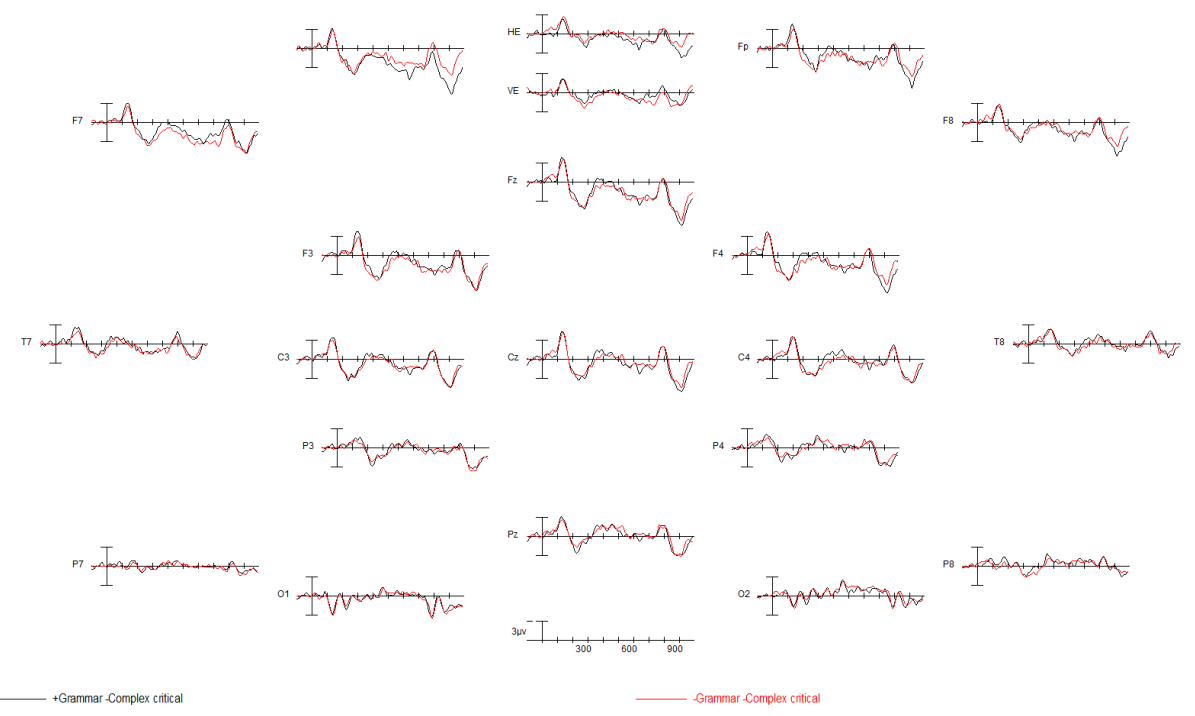

Figure 3: Brain responses in the $100 \mathrm{~ms}$ window following the onset of the critical word in the morphologically simple condition. Grammatical in black, ungrammatical in red. Positive is plotted down

The relationship between the two was not significant $(p=.95)$. Additionally, TLEPT scores did not predict behavioral results as measured by $d^{\prime}(p=.95)$.

\subsection{ERP Findings}

Figure 3 and Figure 4 show the grand mean of responses in the $1000 \mathrm{~ms}$ time window following the erroneous stimulus. Figure 3 shows the difference between grammaticality conditions when the target verb was morphologically simple. Figure 4 shows the difference when the target verb was morphologically complex. As can be seen in both montages, there is no obvious P600 effect.

However, grand mean analyses can obscure subject variances, especially so in a population expected to be highly heterogeneous, like L2 speakers. As introduced in Tanner et al. [17] and Tanner, Inoue, and Osterhout [52], a plot of P600 magnitudes vs N400 magnitudes can serve to visually understand the spread and types of brain responses in the population. To compute these magnitudes, we used six electrodes in the centro-parietal region where N400 and P600 effects are typically largest, over electrodes $\mathrm{C} 3, \mathrm{Cz}, \mathrm{C} 4, \mathrm{P} 3, \mathrm{Pz}$, and P4. N400 magnitude was calculated as the mean activity between $300 \mathrm{~ms}$ and $500 \mathrm{~ms}$ in the grammatical condition minus the ungrammatical condition across both complexity types. P600 magnitude was 


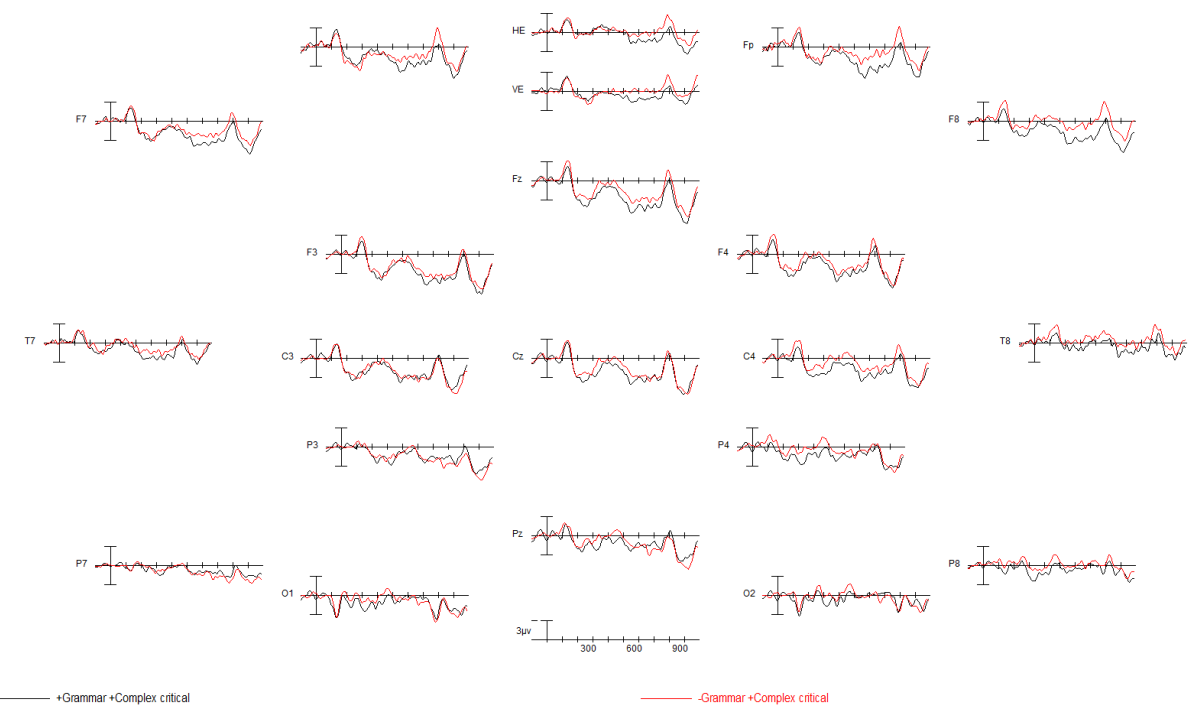

Figure 4: Brain responses in the $100 \mathrm{~ms}$ window following the onset of the critical word in the morphologically simple condition. Grammatical in black, ungrammatical in red. Positive is plotted down

calculated as the mean activity between $500 \mathrm{~ms}$ and $900 \mathrm{~ms}$ in the ungrammatical condition minus the grammatical condition across both complexity types.

Figure 5 shows that roughly half of subjects are still displaying primarily N400 effects (upper-left quadrant) and roughly half are displaying primarily P600 effects (lower-right quadrant). The relationship is highly negatively correlated $(r=-.6591$, $p<.001$ ), which implies as the magnitude of one effect increases, the other decreases, consistent with the literature.

Patterns along this line can be quantified by the Response Dominance Index (RDI) and Response Magnitude Index (RMI) [52]. The RDI quantifies the individual's response dominance (N400 or P600) by finding their least squares distance from an equal effect. RDI values near zero reflect equal-sized N400 and P600 effects, and larger positive values reflect larger P600 effects, and more negative values reflect larger N400 effects. The eight participants with positive RDI values will comprise the subset for the next analysis.

Because the RDI is the same when the two effects are equally sized, no matter their magnitude (i.e. the RDI is the same for two equally large effects as two equally small effects), we additionally calculate the RMI by calculating each individual's Euclidean distance from zero. RMI measures overall sensitivity to violations; larger values indicate greater neural response, representing larger integration difficulty. 


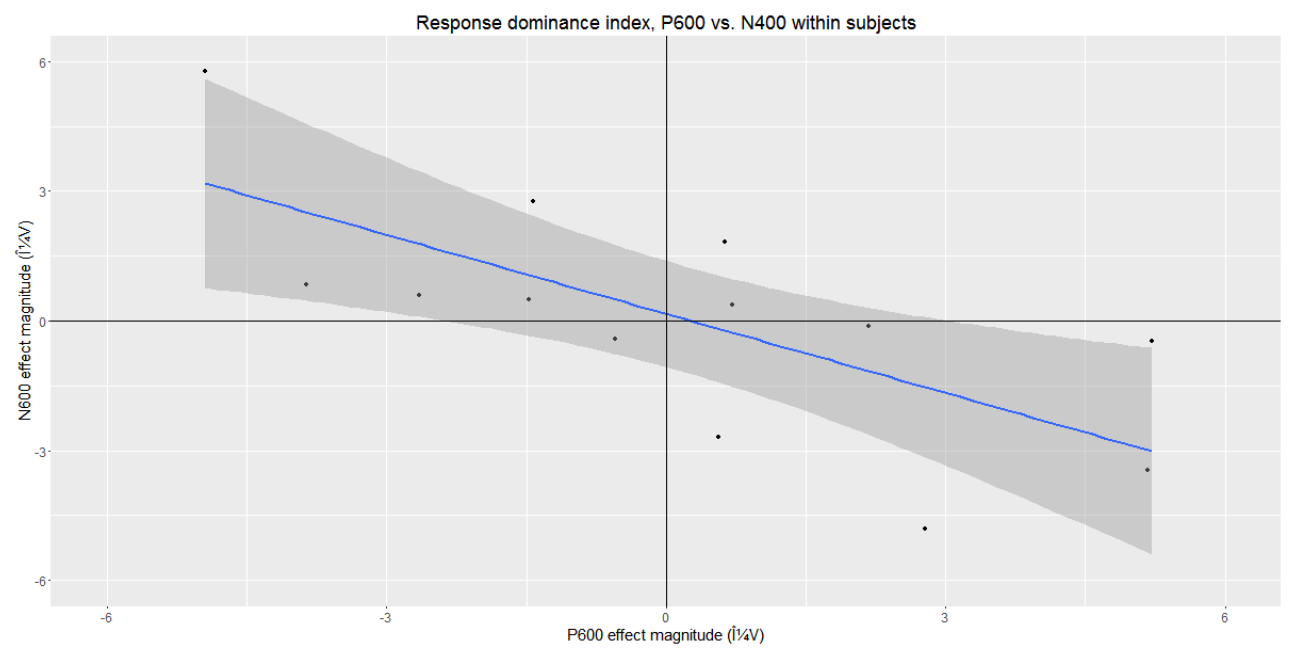

Figure 5: Scatterplot showing N400 magnitudes vs P600 magnitudes over six electrodes demonstrating the effect. Each dot represents a single learner and the line represents the linear regression with a 95\% confidence interval. Participants in the upper left quadrant display a primarily N400 effect and participants in the lower right quadrant display a P600 effect primarily.

We chose to evaluate the effect of frequency, proficiency (TLEPT), accuracy $\left(d^{\prime}\right)$, AoE, and AoA as possible predictors of RDI and RMI. Because AoA is highly rightskewed, we used the logarithm of AoA in the model. Distributional statistics for the dependent variables are given in Table 3. Frequency was rated for both Mandarin and English (and any other languages spoken, when applicable) on a scale of 1-3 across seven communicative domains: employers/teachers, mother/father, brothers/sisters, friends, yourself, classmates/peers, and pets. Frequency used in models was the average of the seven responses.

The full correlation matrix for the dependent variables and the RDI and RMI is given in Table 4. No correlations between predictors and RDI/RMI were significant, although the correlations between $\log (\mathrm{AOA})$ and RDI/RMI approached significance at $p<.05$ (RDI $p=0.079$, RMI $p=0.095)$.

However, a visual examination of the relationship between $\log (\mathrm{Ao} A)$ and RDI/RMI reveals that the possible effect is being driven entirely by one outlier subject who arrived in an English-speaking country at age 4 (Figure 6).

The average AoA was 17.1 with a standard deviation of 1.5. This leaves an AoA of 4 well below the $3 \sigma$ threshold for rejection. In fact, the effect disappears with that subject rejected (RDI $p=0.58$, RMI $p=0.56$ ). 


\begin{tabular}{lrrc}
\hline Measure & Mean & SD & Range \\
\hline RDI & 0.13 & 2.22 & $-3.50-3.69$ \\
RMI & 2.48 & 1.80 & $0.55-5.22$ \\
Freq. & 2.19 & 0.27 & $1.43-2.43$ \\
TLEPT & 0.84 & 0.05 & $0.77-0.94$ \\
d-prime & 1.20 & 0.95 & $-0.01-3.15$ \\
AoE & 6.50 & 2.53 & $2.5-10.0$ \\
$\log ($ AoA $)$ & 1.18 & 0.18 & $0.60-1.30$ \\
\hline
\end{tabular}

Table 3: Mean, standard deviation, and range, for the variables used in regression models.

\begin{tabular}{rrrrrrr}
\hline & AOE.E & freq.E & TLEPT & $\log$ AOA & $d^{\prime}$ & RDI \\
\hline AOE.E & - & & & & & \\
freq.E & -0.13 & - & & & & \\
TLEPT & 0.08 & -0.10 & - & & & \\
$\log$ AOA & 0.06 & 0.02 & 0.44 & - & & \\
$d^{\prime}$ & -0.16 & 0.18 & -0.02 & -0.62 & - & \\
RDI & 0.03 & 0.43 & -0.37 & -0.50 & 0.24 & - \\
\hline
\end{tabular}

Table 4: Correlation matrix for dependent variables used in regression models, $p<$ 0.05

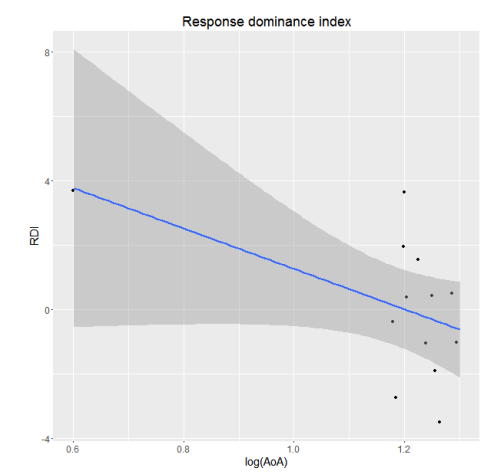

(a) The relationship between RDI and $\log (\mathrm{AoA})$. Positive values indicate a $\mathrm{P} 600$ dominant response and negative values an N400-dominant response. The relationship is not significant.

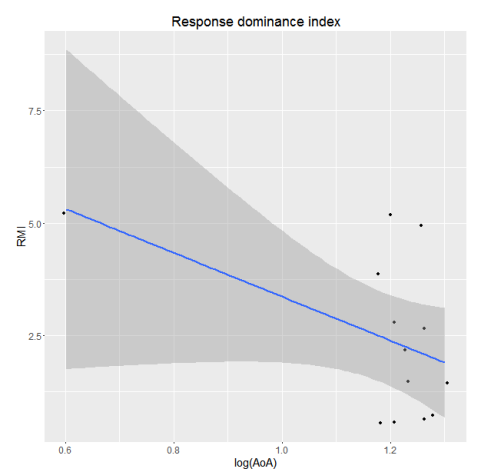

(b) The relationship between RMI and $\log (\mathrm{AoA})$. Larger values indicate a larger magnitude of response The relationship is not significant.

Figure 6: An examination of the relationship between age of arrival and RDI/RMI. 

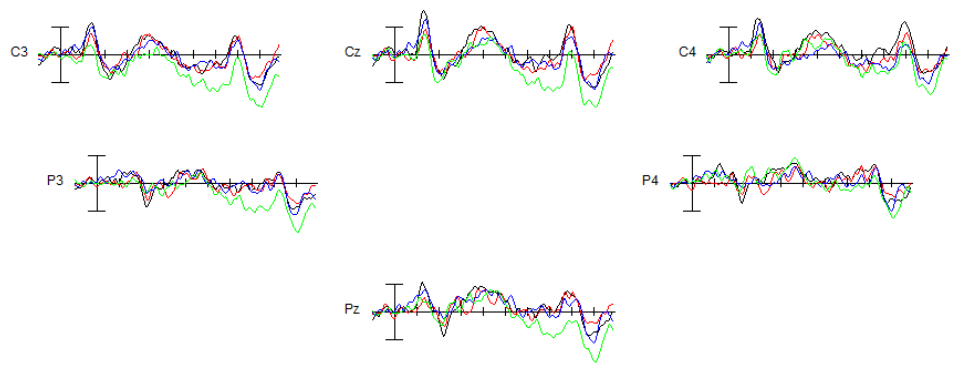

+Grammar -Complex critical

+Grammar +Complex critical

-Grammar-Complex critical

-Grammar + Complex critical

Figure 7: The $1000 \mathrm{~ms}$ time window following the critical word for all four experimental conditions.

\subsection{Complexity Results}

Because the question of this paper is of the behavior of the P600 in L2 speakers, it makes little sense to group subjects without P600-like responses in the analysis. Eight participants had a positive RDI, and they will be used in this subgroup analysis.

For this subgroup, the average age of exposure to English was 6.64 (min: 2.5; max: 10). Both the Japanese-speaking and Bahasa-speaking participants were included in this group.

Figure 7 shows the $1000 \mathrm{~ms}$ window for all four experimental conditions. Using a two-way ANOVA, the effect of grammaticality on the P600 is significant ( $p<$ $.01)$ but complexity is not $(p=.82)$. Using a linear model on the ungrammatical signals only, the P600 in the complex condition is significantly greater than the simple condition $(p=.01)$.

A two-way ANOVA was conducted on accuracy for the P600 subgroup. There was no effect of complexity $(p=.56)$, suggesting even the ostensibly more advanced learners are no better at discriminating present participle conditions. Like the overall group, there was an effect of grammaticality $(p=.047)$.

\section{Discussion}

\subsection{Complexity Results}

Among participants who showed a P600 at all, they showed only a P600 effect to ungrammatical stimuli with morphologically complex critical words (e.g. *"must running”). Ungrammatical stimuli with morphologicaly simple critical words (e.g. * "is run") did not elicit a P600 effect. This was not a result predicted beforehand. It is 


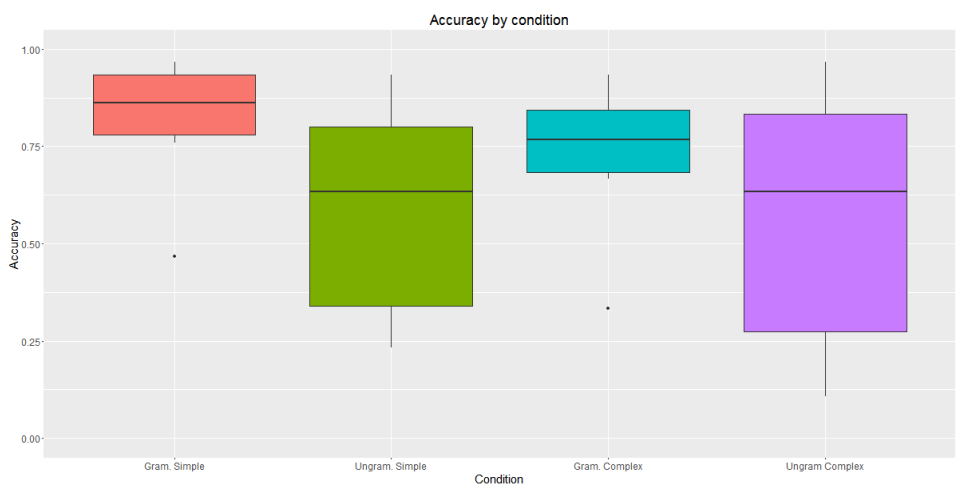

Figure 8: Accuracies by condition for the P600 subgroup

clear that L1 speakers of Mandarin at this ability do not show native-like processing in that they are not sensitive to violations where the critical word is morphologically simple. This suggests an asymmetry in their understanding of the two forms; or possibly an asymmetry in their understanding of the precritical contexts: "be" versus modal. I offer two theoretical explanations for these findings.

(Theory 1) In a predicative model, speakers show P600 responses when a structural or syntactic prediction is violated. Violations of stronger predictions therefore elicit larger P600s. This model suggests native speakers show smaller P600s in the "be" + bare verb condition because there is more heterogeneity in what can follow forms of "be" that lead native speakers to make weaker predictions in that context. As mentioned, a participle follows a form of "be" 13.5 percent of the time. On the other hand, modals have a more uniform context, meaning that unexpected forms of the verb are quite surprising.

The L2 speakers' results are amenable to this theory. With insufficient input, they may not have learned to make predictions about what can follow "be." In fact, non-participle forms of the verb can follow forms of "be" in contexts such as passives, as in (8).

(8) a. The man was run out of town.

b. The company is run by the board.

A lack of a neural response here suggest that learners do not have enough knowledge of "be" to make predictions about it; therefore no predictions are violated when a non-participle form of the verb in encountered.

Despite the fact that modals are less frequent than "be", they are followed by the bare form of the verb 34.6 percent of the time; more frequently than the "be" + bare verb condition, meaning learners may be more willing to make predictions about 
content. This suggests that the threshold for making predictions may lie between 13.5 and 34.6 percent usage of one form over others.

(Theory 2) The second explanation deals with a backward-search perspective. A native speaker may initiate a backward search for the licensor of a feature, and upon not encountering one, respond with a P600 as an indicator of integration difficulty. Because bare forms of the verb are underspecified for a number of features, the lack of a licenser may not be as challenging to integrate for a native speaker compared to a present participle, which is more restricted in context.

It is clear from the findings that the L2 participants do not encounter trouble integrating the bare form of a verb, no matter the context. This means that they have not yet completely learned what the bare form is specified for. On the other hand, present participles are more salient, and learners may have an easier time picking up on the specifications of participles; namely, that they are licensed by auxiliary "be." However, they are seemingly not aware of errors where the licensed participle is missing. To summarize, the learners' active, un-revised rule at this stage states only that present participles must follow "be," rather than stating that auxiliary "be" licenses solely present participles. Such a misunderstanding may also stem from a lack of understanding the difference between auxiliary "be" and the copula.

\subsection{Trends}

Although no correlations were significant, a number of trends are suggestive of relationships between predictors and signal. For example, d-prime was negatively correlated with $\log (\mathrm{AoA})(r=-.62)$, suggesting that later arrivals do less well on the behavioral task. RDI was negatively correlated with $\log (\mathrm{AoA})(r=-0.50)$, suggesting that later arrivals have weaker P600 signals. Frequency of English use was positively correlated with $\mathrm{RDI}(r=.43)$, suggesting that L2 speakers who speak English more often have larger P600 signals. Because of the low sample size for this analysis $(n=7)$, this subgroup analysis has relatively weak power, and I suspect a study with a larger sample size and more distribution on predictors such as AoA may reveal a significant trend.

\subsection{Limitations}

Unlike previous studies, d-prime was not significantly correlated with RDI in this study. I suggest that this may have been because this paradigm could have been especially challenging to L2 speakers due to the length of the stimuli, which were originally designed for native speakers, and even then, were long. 
Secondly, the study was constrained by the small sample size $(n=13)$ and the size of the P600 subgroup $(n=16)$, furthermore, the participants demographic variables were highly clustered around things such as age, AoA, and TLEPT score.

Finally, AoE was self-reported, with a single question. I am concerned participants had different interpretations of "exposed." For example, on participant put down they were exposed to both Mandarin and English at age 3; although presumably they were exposed to at least one from birth. Exposure to one person may be a parent speaking it, attending English class in school, or starting watching English cartoons (for example). A follow-up study should do a more systematic job of characterizing AoE for non-native speakers.

\section{Conclusion}

L2 speakers of English are asymmetrically sensitive to violations of modal/verb structures, but not auxiliary "be"/verb structures. This information suggests that L1 Mandarin speakers at relatively advanced English proficiency know present participles must be licensed by "be," but not that auxiliary "be" obligatorily licenses present participles. 


\section{References}

[1] Eric H. Lenneberg. "The biological foundations of language". In: Hospital Practice 2.12 (1967), pp. 59-67.

[2] Christine M. Weber-Fox and Helen J. Neville. "Maturation constraints on functional specializations for language processing: ERP and behavioral evidence in bilingual speakers". In: Fournal of Cognitive Neuroscience 8.3 (1996), pp. 231-256.

[3] James Emil Flege, Grace H. Yeni-Komshian, and Serena Liu. "Age Constraints on Second-Language Acquisition”. In: fournal of Memory and Language 41 (1991), pp. 78-104.

[4] Sonja A. Kotz. "A critical review of ERP and fMRI evidence on L2 syntactic processing”. In: Brain and Language 109 (2009), pp. 68-74. DOI: 10.1016/j . bandl.2008.06.002.

[5] Kang-Kwong Luke et al. "Functional anatomy of syntactic and semantic processing in language comprehension”. In: Human brain mapping 16.3 (2002), pp. 133-145.

[6] Arturo E. Hernandez, Juliane Hofmann, and Sonja A. Kotz. "Age of acquisition modulates neural activity for both regular and irregular syntactic functions". In: NeuroImage 36.3 (2007), pp. 912-923.

[7] Isabell Wartenburger et al. "Early setting of grammatical processing in the bilingual brain”. In: Neuron 37.1 (2003), pp. 159-170.

[8] Shirley-Ann Rüschemeyer et al. "Processing lexical semantic and syntactic information in first and second language: fMRI evidence from German and Russian”. In: Human brain mapping 25.2 (2005), pp. 266-286.

[9] Shirley-Ann Rüschemeyer, Stefan Zysset, and Angela D. Friederici. "Native and non-native reading of sentences: An fMRI experiment". In: NeuroImage 31.1 (2006), pp. 354-365.

[10] Karsten Steinhauer. "Event-related Potentials (ERPs) in Second Language Research: A Brief Introduction to the Technique, a Selected Review, and an Invitation to Reconsider Critical Periods in L2". In: Applied Linguistics 35.4 (2014), p. 393. DOI: 10.1093/applin/amu028. eprint: /oup/backfile/Content_ public/Journal/applij/35/4/10 .1093/applin/amu028/2/amu028. pdf. URL: +\%20http://dx. doi .org/10.1093/applin/amu028.

[11] Johanna M. Zumer et al. "A probabilistic algorithm integrating source localization and noise suppression for MEG and EEG data”. In: NeuroImage 37.1 (2007), pp. 102-115. 
[12] Lee Osterhout and Phillip J. Holcomb. "Event-related potentials and syntactic anomaly: Evidence of anomaly detection during the perception of continuous speech”. In: Language and Cognitive Processes 8 (1993), pp. 413-437.

[13] Mark Allen, William Badecker, and Lee Osterhout. "Morphological analysis in sentence processing: an ERP study". In: Language and Cognitive Processes 18 (2011), pp. 405-430.

[14] Alison S. Mehravari et al. "Effects of Grammaticality and Morphological Complexity on the P600 Event-Related Potential Component". In: PLoS ONE 10.10 (2015), e0140850. Dor: 10.1371/journal . pone. 0140850.

[15] Angela D. Friederici. "Towards a neural basis of auditory sentence processing”. In: Trends in cognitive sciences 6.2 (2002), pp. 78-84.

[16] Karsten Steinhauer and John E. Drury. "On the early left-anterior negativity (ELAN) in syntax studies”. In: Brain and language 120.2 (2012), pp. 135-162.

[17] Darren Tanner et al. "Individual differences reveal stages of L2 grammatical acquisition: ERP evidence". In: Bilingualism: Language and Cognition (16 2012), pp. 367-382.

[18] Darren Tanner, Janet Nicol, and Laurel Brehm. "The time-course of feature intereference in agreement comprehension: Multiple mechanisms and asymmetrical attration". In: Journal of Memory and Language 76 (2014), pp. 195215. DoI: $10.1016 / j \cdot j m l .2014 .07 .003$.

[19] Lee Osterhout, Judith McLaughlin, and Micheal Bersick. "Event-related brain potential and human language”. In: Trends in Cognitive Sciences 1.6 (Sept. 1997), pp. 203-209.

[20] Marta Kutas and Kara D. Federmeier. "Thirty years and counting: Finding meaning in the N400 component of the event-related brain potential (ERP)". In: Annual Review of Psychology 62 (2011), pp. 621-647.

[21] Peter Hagoort, Colin M. Brown, and Jolanda Groothusen. "The syntactic positive shift as an ERP measure of syntactic processing”. In: Language and Cognitive Processes 8 (1993), pp. 439-484.

[22] Lee Osterhout and Janet Nicol. "On the distinctiveness, independence, and time course of the brain responses to syntactic and semantic anomalies". In: Language and Cognitive Processes 14 (3 1999), pp. 283-317.

[23] Angela D. Friederici, Anja Hahne, and Axel Mecklinger. "Temporal structure of syntactic processing: Early and late event-related potential Effects". In: Journal of Experimental Psychology: Language, Memory and Cognition 22 (1996), pp. 1219-1248. 
[24] Edith Kaan and Tamara Y. Swaab. "Repair, revision and complexity in syntactic analysis: An electrophysiological differentiation". In: fournal of Cognitive Neuroscience 15.98-110 (2003).

[25] Lee Osterhout and Linda A. Mobley. "Event-related brain potentials elicited by failure to agree". In: fournal of Memory and Language 34 (1995), pp. 739773.

[26] Lee Osterhout, Philip J. Holcomb, and David A. Swinney. "Brain potentials elicited by garden-path sentences: Evidence for the application of verb information during parsing”. In: Journal of Experimental Psychology: Language, Memory and Cognition 28 (1995), pp. 786-803.

[27] Nicola Molinaro, Horacio A. Barber, and Manuel Carreiras. "Grammatical agreement processing in reading: ERP findings and future directions". In: Cortex 47 (2011), pp. 908-930. DoI: 10.1016/j . cortex.2011.02.019.

[28] Aniruddh D. Patel et al. "Processing Syntactic Relations in Language and $\mathrm{Mu}-$ sic: An Event-Related Potential Study”. In: Journal of Cognitive Neuroscience 10.6 (1998), pp. 717-733.

[29] Cara R. Featherstone et al. "Semantics, Syntax or Neither? A Case for Resolution in the Interpretation of N500 and P600 Responses to Harmonic Incongruities". In: PLoS ONE (2013). DoI: 10.1371/journal . pone. 0076600.

[30] Lee Osterhout and Phillip J. Holcomb. "Event-related brain potentials elicited by syntactic anomaly”. In: fournal of Memory and Language 31.6 (1992), pp. 785806.

[31] Edith Kaan et al. "The P600 as an index of syntactic integration difficulty". In: Language and Cognitive Processes 15.2 (2000), pp. 159-201. Dor: 10.1080 / 01690960036084.

[32] Colin Phillips, Nina Kazanina, and Shani H. Abada. "ERP effects of the processing of syntactic long-distance dependencies". In: Cognitive Brain Research 22.3 (2005), pp. 407-428. IssN: 0926-6410. Dor: http: //doi .org/10.1016/ j. cogbrainres.2004.09.012. uRL: http: //wWw.sciencedirect.com/ science/article/pii/S0926641004002526.

[33] Stefan Frisch et al. "The P600 as an indicator of syntactic ambiguity". In: Cognition 85 (2002), B83-B92.

[34] Aangela D. Friederici et al. "Syntactic parsing preferences and their on-line revisions: a spatio-temporal analysis of event-related brain potentials". In: Cognitive Brain Research 11 (2 2001), pp. 305-323. DoI: 10 . 1016/ S0926 $6410(00) 00065-3$. 
[35] Mark Davies. The Corpus of Contemporary American English: 450 million words, 1990-present. 2008.

[36] Jennifer M. Goldschneider and Robert M. DeKeyser. "Explaining the 'Natural Order' of L2 Morpheme Acquisition in English: A Meta-analysis of Multiple Determinants”. In: Language Learning 51.1 (2001), pp. 1-50.

[37] Marta Kutas and Steven A. Hillyard. "Event-related brain potentials to semantically inappropriate and surprisingly large words”. In: Biological psychology 11.2 (1980), pp. 99-116.

[38] Sten Ardal et al. "Brain responses to semantic incongruity in bilinguals". In: Brain and language 39.2 (1990), pp. 187-205.

[39] Anja Hahne. "What's different in second-language processing? Evidence from event-related brain potentials". In: fournal of psycholinguistic research 30.3 (2001), pp. 251-266.

[40] Shiro Ojima, Hiroki Nakata, and Ryusuke Kakigi. "An ERP study of second language learning after childhood: Effects of proficiency”. In: Journal of Cognitive Neuroscience 17.8 (2005), pp. 1212-1228.

[41] Sonja Rossi et al. "The Impact of Proficiency on Syntactic Second-language Processing of German and Italian: Evidence from Event-related Potentials". In: Journal of Cognitive Neuroscience 18.12 (2006), pp. 203-2048.

[42] Anja Hahne and Angela D Friederici. "Processing a second language: Late learners' comprehension mechanisms as revealed by event-related brain potentials”. In: Bilingualism: Language and Cognition 4.02 (2001), pp. 123-141.

[43] Judith McLaughlin et al. "Brain potentials reveal discrete stages of L2 grammatical learning”. In: Language Learning 60.s2 (2010), pp. 123-150.

[44] Laura Sabourin and Laurie A. Stowe. "Second language processing: when are first and second languages processed similarly?” In: Second Language Research 24.3 (2008), pp. 397-430.

[45] Cheryl Frenck-Mestre et al. "Processing of grammatical gender in French as a first and second language: Evidence from ERPs". In: Eurosla yearbook 9.1 (2009), pp. 76-106.

[46] Kara Morgan-Short et al. "Second Language Processing Shows Increased NativeLike Neural Responses after Months of No Exposure”. In: PLoS ONE (2012). DOI: 10.1371 /journal. pone.0032974. 
[47] Kara Morgan-Short et al. "Explicit and Implicit Second Language Training Differentially Affect the Achievement of Native-like Brain Activation Patterns”. In: Journal of Cognitive Neuroscience 24.4 (2012), pp. 933-947. DOI: 10.1162/jocn_a_00119.

[48] Michael T. Ullman. "The declarative/procedural model of lexicon and grammar”. In: fournal of Psycholinguistic Research 30.1 (2001), pp. 37-69.

[49] Kirsten Weber and Aureliu Lavric. "Syntactic anomaly elicits a lexico-semantic (N400) ERP effect in the second language but not the first". In: Psychophysiology 45.6 (2008), pp. 920-925.

[50] R.H. Baayen, R. Piepenbrock, and L. Gulikers. The CELEX lexical database. CD-ROM. 1995.

[51] Herbert H. Jasper. "The ten-twenty system of the International Federation". In: Electroencephalography Clinical Neurophysiology 10 (1958), pp. 371-375.

[52] Darren Tanner, K. Inoue, and Lee Osterhout. "Brain-based individual differences in online L2 grammatical comprehension”. In: Bilingualism: Language and Cognition (2013), pp. 1-17. DOI: 10.1017/S1366728913000370. 